\title{
NON-CONJUGATE OSCULATING QUADRICS OF A CURVE ON A SURFACE*
}

\author{
BY \\ R. C. BULLOCK
}

1. Introduction. This paper is concerned with making a study of the projective differential geometry of a non-conjugate net of curves on a surface in three-space by means of a pair of osculating quadrics defined in the following manner. Consider a curve $C$ on the surface $S$. At a point $P$ of $C$ and at two neighboring points $P_{1}, P_{2}$ on $C$ construct the tangents of the curves of one family of the non-conjugate net. The limit of the quadric surface determined by these three lines as the points $P_{1}, P_{2}$ approach $P$ along $C$ is a non-conjugate osculating quadric at the point $P$ on $C$. The other osculating quadric is obtained in a similar manner by drawing tangents to the other family of the net. Thus we associate with each point of the surface a pair of osculating quadrics analogous to the asymptotic osculating quadrics $\dagger$ of Bompiani and Klobouček and the conjugate osculating quadrics $\ddagger$ of Lane.

We compute the equations of the osculating quadrics and note some results that follow rather immediately therefrom. The chief contribution of the paper is a complete discussion of the nature of the curve of intersection of the two osculating quadrics at a point of a curve on the surface. We also study the curve of intersection of corresponding osculating quadrics for two curves which are respectively members of two families of curves which form a conjugate net on the surface. As a by-product we obtain a new necessary and sufficient condition that a net of curves on the surface be a conjugate net.

2. Analytic basis. In this section we set up an analytic basis for the study of a non-conjugate net of curves on a surface in projective three-space following Green's method. $\$$ We shall also list in this section certain of Green's results that we shall need for later reference. Let it be noted here that we shall assume that the surface sustaining the net is not developable, and that unless otherwise specifically stated the net in question is not the asymptotic net.

\footnotetext{
* Presented to the Society, December 29, 1932; received by the editors August 25, 1932.

$\dagger$ E. P. Lane, Projective Differential Geometry of Curves and Surfaces, University of Chicago Press, 1932, p. 80.

$\ddagger$ E. P. Lane, Conjugate nets and the lines of curvature, American Journal of Mathematics, vol. 53 (1931), p. 577.

§ G. M. Green, Nets of space curves, these Transactions, vol. 21 (1920), pp. 207-236.
} 
Let the surface under consideration be the analytic surface whose vector equation in homogeneous coordinates is

$$
x=x(u, v) .
$$

A necessary and sufficient condition that the parametric curves $u=$ const., $v=$ const. shall not form a conjugate net is that $x$ shall not satisfy an equation of Laplace of the form

$$
a x_{u v}+b x_{u}+c x_{v}+d x=0 .
$$

This is equivalent to saying that the fourth-order determinant

$$
W=\left(x_{u v}, x_{u}, x_{v}, x\right)
$$

does not vanish. Hence the functions $x$ are solutions of a pair of partial differential equations of the form

$$
x_{u u}=p x+\alpha x_{u}+\beta x_{v}+L x_{u v}, \quad x_{v v}=q x+\gamma x_{u}+\delta x_{v}+N x_{u v} .
$$

If we adjoin certain integrability conditions, which we shall not write down, these equations will form a completely integrable system; that is, any fundamental set of solutions can be expressed as a linear combination, with constant coefficients, of any other fundamental system. We shall base our projective theory of surfaces on this completely integrable system of partial differential equations.

If we regard the $u$-tangent at a point $P_{x}$ as a generator of the congruence of $u$-tangents, its other focal point is given by

$$
\rho=x_{u}+(\beta / L) x \text {. }
$$

Similarly the focal point of the $v$-tangent at $P_{x}$ is given by

$$
\sigma=x_{v}+(\gamma / N) x .
$$

The line joining $\rho$ and $\sigma$ is called by Green the ray of the point $P_{x}$.

Since the parametric net is not asymptotic the osculating planes of the $u$-curve and $v$-curve through $P_{x}$ determine a line that passes through $P_{x}$ but does not lie in the tangent plane. This line is called by Green the axis of the point $P_{x}$. The totality of axes of points of the surface generate the axis congruence. The point $\tau$ which is the harmonic conjugate of the point $x$ with respect to the two focal points of the axis is given by

$$
\tau=K^{\prime} x+(\gamma / N) x_{u}+(\beta / L) x_{v}+x_{u v},
$$

where the value of $K^{\prime}$ is given by

$$
\begin{aligned}
K^{\prime}= & -\frac{1}{2}\left[b^{(21)}+c^{(12)}-(\beta / L) a^{(12)}-(\gamma / N) a^{(21)}+(\alpha \gamma) / N+(\beta \delta) / L\right. \\
& -L \gamma^{2} / N^{2}-N \beta^{2} / L^{2}-2 \beta \gamma /(L N)+\left(N \gamma_{u}-N_{u} \gamma\right) / N^{2} \\
& \left.+\left(L \beta_{v}-L_{v} \beta\right) / L^{2}\right],
\end{aligned}
$$


wherein

$$
\begin{aligned}
& a^{(21)}=\left[L_{v}+\alpha+\beta N+L\left(N_{u}+\gamma L+\delta\right)\right] /(1-L N), \\
& a^{(12)}=\left[N\left(L_{v}+\alpha+\beta N\right)+N_{u}+\gamma L+\delta\right] /(1-L N), \\
& b^{(21)}=\left[\alpha_{v}+\beta \gamma+L\left(\alpha \gamma+\gamma_{u}+q\right)\right] /(1-L N), \\
& c^{(12)}=\left[N\left(\beta \delta+\beta_{v}+p\right)+\beta \gamma+\delta_{u}\right] /(1-L N) .
\end{aligned}
$$

The points $\rho, \sigma, \tau$ are covariant points with respect to all transformations of proportionality factor and independent variable in equations (1) that preserve the net.

The curvilinear differential equation defining the asymptotic net on the surface is

$$
L d u^{2}+2 d u d v+N d v^{2}=0 .
$$

Evidently $L=N=0$ is a necessary and sufficient condition that the parametric net be asymptotic. We also see that the surface is developable if, and only if, $1-L N=0$.

In order that the net defined by the equation

$$
(d v-\lambda d u)(d v-\mu d u)=0
$$

shall be a conjugate net the two directions $\lambda$ and $\mu$ must separate harmonically the asymptotic directions. A necessary and sufficient condition for this is

$$
\mu=-(L+\lambda) /(N \lambda+1) \text {. }
$$

The conjugate net whose directions separate harmonically the parametric directions is called by Green the associate conjugate net, and it is defined by the following equation:

$$
L d u^{2}-N d v^{2}=0 .
$$

We list for reference the following invariants due to Green and Grove:*

$$
\begin{aligned}
r & =L+\lambda, s=N \lambda+1, \\
t & =L \lambda^{\prime}-L^{\prime} \lambda, \pi=c_{v}-b_{1 u}, \\
h^{\prime} & =q+b_{1}\left(\delta-b_{1}\right)-b_{1 v}+N b_{1 u}, \\
k & =p+c(\alpha-c)-c_{u}+L c_{v}, \\
B & =N c-\frac{1}{2}\left(a^{(12)}-\delta\right), \\
5^{\prime} & =L b_{1}-\frac{1}{2}\left(a^{(21)}-\alpha\right), \\
G & =c^{(21)}+c a^{(21)}-c_{u}-c^{2}, \\
G^{\prime} & =b^{(12)}+b_{1} a^{(12)}-b_{1 v}-b_{1}^{2}, \\
F & =c^{(12)}+c a^{(12)}-c \delta-N c^{2}-2 c_{v}, \\
F^{\prime} & =b^{(21)}+b_{1} a^{(21)}-b_{1} \alpha-L b_{1}^{2}-2 b_{1 u}, \\
W^{(u)} & =F-N G,
\end{aligned}
$$

* V. G. Grove, $A$ theory of a general net on a surface, these Transactions, vol. 28 (1926), p. 496. 
wherein $a^{(21)}, a^{(12)}, b^{(21)}, c^{(12)}$ are given by (6) and where

$$
\begin{aligned}
a & =1 / L, \quad b=-\alpha / L, \quad c=-\beta / L, \quad d=-p / L, \\
a_{1} & =1 / N, \quad b_{1}=-\gamma / N, \quad c_{1}=-\delta / N, \quad d_{1}=-q / N, \\
b^{(12)} & =\left[N\left(\alpha_{v}+\beta \gamma\right)+\alpha \gamma+\gamma_{u}+q\right] /(1-L N), \\
c^{(21)} & =\left[\beta \delta+\beta_{v}+p+L\left(\beta \gamma+\delta_{u}\right)\right] /(1-L N) .
\end{aligned}
$$

3. The non-conjugate osculating quadrics. We now compute the equations of the quadrics defined in $\$ 1$. Let us consider a non-conjugate net $N$ on a surface $S$ of the kind specified in $\S 2$, and suppose $S$ is an integral surface of the system (1). On this surface let us consider a curve $C_{\lambda}$ which is a member of the family defined by the equation

$$
d v-\lambda d u=0
$$

and suppose this family of curves is not conjugate to either family of the net $N$. We shall first find the equation of the osculating quadric $Q_{u}$ determined by the tangents to three consecutive $u$-curves at a point $P_{x}$ of $C_{\lambda}$. The equation will first be referred to the local tetrahedron of reference whose vertices are the points $x, x_{u}, x_{v}, x_{u v}$. We may define any point $X$ on $C_{\lambda}$ near $P_{x}$ by the following power series in $\Delta u$ :

$$
X=x+x^{\prime} \Delta u+\frac{x^{\prime \prime} \Delta u^{2}}{2} \cdots \quad\left(x^{\prime}=\frac{d x}{d u}\right)
$$

By making use of the differential equations (1) and expressions obtained from them by differentiation this series can be expressed in the form

$$
X=x_{1} x+x_{2} x_{u}+x_{3} x_{v}+x_{4} x_{u v},
$$

where, for a suitably chosen unit point, $x_{1}, x_{2}, x_{3}, x_{4}$ are local coordinates of the point $X$ referred to the local tetrahedron $x, x_{u}, x_{v}, x_{u v}$, and the power series defining them sufficiently far for the purposes of this paper are

$$
\begin{aligned}
& x_{1}=1+\cdots, \\
& x_{2}=\Delta u+\cdots, \\
& x_{3}=\lambda \Delta u+\left(\beta+\lambda^{\prime}+\lambda^{2} \delta\right) \Delta u^{2} / 2+\cdots, \\
& x_{4}=\left(L+2 \lambda+N \lambda^{2}\right) \Delta u^{2} / 2+\cdots .
\end{aligned}
$$


Similarly for a point $X_{u}$ on the $u$-tangent near $P_{x}$ we get the local power series expansions

$$
\begin{aligned}
y_{1}= & p \Delta u+\cdots, \\
y_{2}= & +\alpha \Delta u+\cdots, \\
y_{3}= & \beta \Delta u+\left[\beta_{u}+\alpha \beta+(L+2 \lambda) c^{(21)}\right. \\
& \left.+\lambda^{2} c^{(12)}\right] \Delta u^{2} / 2+\cdots, \\
y_{4}= & (L+\lambda) \Delta u+\left[\lambda^{\prime}+L_{u}+\beta+\alpha L\right. \\
& \left.+(L+2 \lambda) a^{(21)}+\lambda^{2} a^{(12)}\right] \Delta u^{2} / 2+\cdots .
\end{aligned}
$$

Now let us set

$$
\begin{aligned}
& e_{1}=\left(\beta+\lambda^{\prime}+\lambda^{2} \delta\right) / 2, \\
& e_{2}=\left(L+2 \lambda+N \lambda^{2}\right) / 2, \\
& e_{3}=\left[\beta_{u}+\alpha \beta+(L+2 \lambda) c^{(21)}+\lambda^{2} c^{(12)}\right] / 2, \\
& e_{4}=\left[\lambda^{\prime}+L_{u}+\beta+\alpha L+(L+2 \lambda) a^{(21)}+\lambda^{2} a^{(12)}\right] / 2 .
\end{aligned}
$$

Any point on the line $X X_{u}$ is given by the linear combination

$$
m X+n X_{u} \quad(m, n \text { scalars }) .
$$

On making use of equations (12) and (13) the power series expansions for the local coordinates of the point are found to be

$$
\begin{aligned}
& z_{1}=m+p n \Delta u+\cdots, \\
& z_{2}=n+(m+\alpha n) \Delta u+\cdots, \\
& z_{3}=(\lambda m+\beta n) \Delta u+\left(e_{1} m+e_{3} n\right) \Delta u^{2}+\cdots, \\
& z_{4}=r n \Delta u+\left(e_{2} m+e_{4} n\right) \Delta u^{2}+\cdots .
\end{aligned}
$$

Now demand that the power series (14) satisfy the most general equation of a quadric surface identically in $m, n$ and identically in $\Delta u$ as far as terms of the second degree. The computation involved in doing this is rather laborious, and the details will be omitted here. The equation of the quadric $Q_{u}$ is found to be

$$
\left(L-N \lambda^{2}\right) x_{3}{ }^{2}+R x_{3} x_{4}+S^{\prime} x_{4}{ }^{2}+2 \lambda\left(\lambda x_{1} x_{4}-r x_{2} x_{3}+\beta x_{2} x_{4}\right)=0,
$$

where $r$ was given in equations (10) and $R$ and $S^{\prime}$ are given by

$$
\begin{aligned}
R & =2 \lambda\left(\complement^{\prime}-L b_{1}\right)+2 \lambda^{2}(\mathscr{B}-N c)+\left[\lambda^{\prime}-2 \beta-\lambda(\log r)^{\prime}\right], \\
S^{\prime} & =\left(\beta^{2}+\lambda \beta^{\prime}-\alpha \beta \lambda-p \lambda^{2}-\beta \lambda^{\prime}\right) / r+\lambda\left[c^{(21)}+\lambda c^{(12)}\right] .
\end{aligned}
$$

The equation of the osculating quadric $Q_{v}$ may be obtained immediately from that of $Q_{u}$ by performing in equation (15) the following substitution: 


$$
\left(\begin{array}{cccccccc}
u & x_{2} & \alpha & \beta & p & L & \lambda & \lambda^{\prime} \\
v & x_{3} & \delta & \gamma & q & N & 1 / \lambda & -\lambda^{\prime} / \lambda^{3}
\end{array}\right) .
$$

The resulting equation of $Q_{v}$ is

$$
\left(L-N \lambda^{2}\right) x_{2}^{2}+P x_{2} x_{4}+Q x_{4}^{2}-2\left(x_{1} x_{4}-s x_{2} x_{3}+\gamma \lambda x_{3} x_{4}\right)=0,
$$

where $s$ was given in equations (10) and $P$ and $Q$ are defined by

$$
\begin{aligned}
& P=2 \gamma \lambda^{2}+(\log s)^{\prime}-2\left(\mathbb{C}^{\prime}-L b_{1}\right)-2 \lambda(\mathfrak{B}-N c), \\
& Q=\lambda\left[\delta \gamma \lambda-\gamma^{\prime}+q-\gamma(\log \lambda)^{\prime}-\gamma^{2} \lambda^{2}\right] / s-\left(b^{(21)}+\lambda b^{(12)}\right) .
\end{aligned}
$$

If we specialize our net to be the asymptotic net by putting $L=N=0$, equations (15) and (17) reduce to the equations for the asymptotic osculating quadrics of Bompiani and Klobouček.

4. A covariant tetrahedron of reference. The tetrahedron to which the equations of the osculating quadrics were referred in the last section was not a covariant tetrahedron, and hence we were unable to express the coefficients in the equations completely in terms of invariants. We find it advantageous to change our tetrahedron of reference to a new covariant tetrahedron whose vertices are the points $x, \rho, \sigma, \tau$, of which the lạst three were defined by equations (2), (3), (4) of $\S 2$. The transformation that effects this change of reference system is

$$
\begin{aligned}
& x_{1}=y_{1}+(\beta / L) y_{2}+(\gamma / N) y_{3}+K^{\prime} y_{4}, \\
& x_{2}=y_{2}+(\gamma / N) y_{4}, \\
& x_{3}=y_{3}+(\beta / L) y_{4}, \\
& x_{4}=y_{4},
\end{aligned}
$$

where the $x$ 's are the old variables and the $y$ 's are the new. After renaming the variables so that the $x$ 's are the new local coordinates, the transformed equations of $Q_{u}$ and $Q_{v}$ are respectively

$$
\begin{aligned}
\left(L-N \lambda^{2}\right) x_{3}{ }^{2} & +\left(2 \lambda 2 \mathscr{B}+2 \lambda \mathbb{C}^{\prime}+t / r\right) x_{3} x_{4}+\lambda\left(G-\lambda F^{\prime}\right. \\
& +\lambda \pi-\lambda k / r) x_{4}{ }^{2}+2 \lambda^{2} x_{1} x_{4}-2 \lambda r x_{2} x_{3}=0, \\
\left(L-N \lambda^{2}\right) x_{2}{ }^{2} & -\left[2 \lambda \mathscr{B}+2 \mathbb{C}^{\prime}-(\log s)^{\prime}\right] x_{2} x_{4}+\left(F-\lambda G^{\prime}\right. \\
& \left.+\lambda \pi+\lambda h^{\prime} / s\right) x_{4}{ }^{2}-2 x_{1} x_{4}+2 s x_{2} x_{3}=0 .
\end{aligned}
$$

The coefficients in the equations of the osculating quadrics are now expressed completely in terms of invariants defined in (10).

5. Some immediate results. In this section we establish some theorems 
analogous to those of Lane for a conjugate net and in addition obtain an interesting reciprocity property.

The osculating quadric $Q_{u}$ intersects the tangent plane, $x_{4}=0$, in the $u$ tangent, $x_{3}=x_{4}=0$, and the residual line

$$
x_{4}=\left(L-N \lambda^{2}\right) x_{3}-2 \lambda r x_{2}=0 .
$$

This line is the $v$-tangent if, and only if, $L-N \lambda^{2}=0$. Likewise $Q_{v}$ intersects the tangent plane in the $v$-tangent, $x_{2}=x_{4}=0$, and the residual line

$$
x_{4}=\left(L-N \lambda^{2}\right) x_{2}+2 s x_{3}=0 .
$$

On referring to equation (9) we conclude the following result:

$A$ necessary and sufficient condition that a curve on our surface belong to the associate conjugate net is that either of the two osculating quadrics have the tangents of the net as generators at every point of the curve.

Now suppose $L-N \lambda^{2} \neq 0$. Then the two lines (19) and (20) coincide at every point of $C_{\lambda}$ if, and only if, equation (7) holds. Thus we have the following theorem:

The residual intersections of the osculating quadrics with the tangent plane coincide at every point of a curve if, and only if, the curve is a member of one family of the asymptotic net on the surface. The line of coincidence is the tangent to the curve.

The coordinates $\xi$ of the polar plane of any point $x$ with respect to the osculating quadric $Q_{u}$ are given by

$$
\begin{aligned}
\xi_{1}= & 2 \lambda^{2} x_{4}, \quad \xi_{2}=-2 \lambda r x_{3}, \\
\xi_{3}= & -2 \lambda r x_{2}+2\left(L-N \lambda^{2}\right) x_{3} \\
& +\left(2 \lambda^{2} \mathscr{B}+2 \lambda \complement^{\prime}+t / r\right) x_{4}, \\
\xi_{4}= & 2 \lambda^{2} x_{1}+\left(2 \lambda^{2} \mathscr{B}+2 \lambda\left(\complement^{\prime}+t / r\right) x_{3}\right. \\
& +2 \lambda\left(G-\lambda F^{\prime}+\lambda \pi-\lambda k / r\right) x_{4} .
\end{aligned}
$$

The polar plane of the point $\rho$, whose local coordinates are $(0,1,0,0)$, with respect to $Q_{u}$ is the plane $x_{3}=0$, which is the osculating plane to the $u$-curve $C_{u}$ at $P_{x}$. But we see from the first of equations (18) that $\rho$ lies on $Q_{u}$, hence we conclude that the osculating plane to the curve $C_{u}$ at a point of a curve $C_{\lambda}$ is tangent to the corresponding osculating quadric $Q_{u}$ at the point $\rho$. In a similar manner it may be shown that the osculating plane to $C_{v}$ at $P_{x}$ is tangent to $Q_{v}$ at the point $\sigma$.

By referring to (21) we see that the polar plane of the point $\sigma$ with respect to $Q_{u}$ passes through the point $P_{x}$, and it passes also through the point $\tau$ if, and only if, 


$$
2 \lambda^{2} \mathscr{B}+2 \lambda \mathfrak{S}^{\prime}+t / r=0 .
$$

We readily draw the following conclusion:

Condition (22) is necessary and sufficient for the ray and axis to be reciprocal polar lines with respect to the quadric $Q_{u}$.

Similar results hold for the quadric $Q_{v}$.

6. Nature of the intersection of the osculating quadrics. We now make a complete study of the nature of the curve of intersection of the osculating quadrics $Q_{u}$ and $Q_{v}$. This study is made by means of the elementary divisors of the matrix of the pencil of quadrics based on $Q_{u}$ and $Q_{v}$. The method is to compute the elementary divisors, write down the characteristic, and ascertain the general nature of the intersection by referring to results tabulated by Snyder and Sisam.*

For the sake of brevity let us write equations (18) for the osculating quadrics $Q_{u}$ and $Q_{v}$ in the following form:

$$
\begin{aligned}
& A x_{3}^{2}+2 B x_{3} x_{4}+C x_{4}^{2}+2 \lambda^{2} x_{1} x_{4}-2 \lambda r x_{2} x_{3}=0, \\
& A x_{2}^{2}-2 D x_{2} x_{4}+E x_{4}^{2}-2 x_{1} x_{4}+2 s x_{2} x_{3}=0,
\end{aligned}
$$

where $A, B, C, D, E$ are new notations introduced here, and their values may be readily read off from (18). We now give the results obtained for the various cases that arise in our problem.

Case I. If $A \neq 0, B-D \lambda \neq 0, L+2 \lambda+N \lambda^{2} \neq 0$, we find that the characteristic is [13]. Hence the intersection of the quadrics $Q_{u}$ and $Q_{v}$ at each point of a curve $C_{\lambda}$ satisfying the conditions stated is a quartic curve with a cusp. But if $B-D \lambda=0$ we get by using the values of $B$ and $D$

$$
t / r+\lambda(\log s)^{\prime}=0
$$

and

$$
t / r=\left(L \lambda^{\prime}-L^{\prime} \lambda\right) /(L+\lambda)=\lambda^{\prime}-\lambda[\log (L+\lambda)]^{\prime} .
$$

Substituting this in (24), dividing by $\lambda$, and integrating we get

$$
\lambda(N \lambda+1) /(L+\lambda)=e \quad(e=\text { const. }) .
$$

Now by replacing $\lambda$ by $d v / d u$ we see that if $C_{\lambda}$ is a curve such that $B-D \lambda=0$ then $C_{\lambda}$ belongs to the pencil of families of curves defined by the curvilinear differential equation

$$
e L d u^{2}+(e+1) d u d v+N d v^{2}=0 .
$$

But let us note that for $e=-1$ this becomes $L-N \lambda^{2}=0$, and for $e=+1$ it

* V. Snyder and C. H. Sisam, A nalytic Geometry of Space, New York, 1914, p. 163. 
becomes $L+2 \lambda+N \lambda^{2}=0$ after dividing through by $d u^{2}$. Thus the three conditions imposed at the beginning of this case are all implied by the second. Since in later work no other case arises in which the curve of intersection of the two quadrics is non-degenerate, we state the following theorem:

The two osculating quadrics at each point of a curve on our net intersect in a non-composite quartic curve if, and only if, the curve does not belong to the pencil of families of curves defined by equation (25). The quartic has a cusp at the point $P_{x}$.

In our general treatment no case arises in which at each point of a curve on our net the total intersection of the pair of osculating quadrics contains as a part of it a non-degenerate cubic.

Case II (a). Suppose $B-D \lambda=0, A \neq 0, L+2 \lambda+N \lambda^{2} \neq 0, A\left(C+E \lambda^{2}\right)$ $-B^{2} \neq 0$. We get the characteristic [1(21)], which shows that the curve of intersection is composed of two conics which touch each other. The conics touch at $P_{x}$ and lie in the planes whose equations are

$$
A \lambda\left(x_{3}-\lambda x_{2}\right)+\left[B \lambda \pm\left(B^{2}-A\left(C+E \lambda^{2}\right)\right)^{1 / 2}\right] x_{4}=0 .
$$

Case II (b). If in II(a) we have $A\left(C+E \lambda^{2}\right)-B^{2}=0$, then the characteristic is [1(111)], and hence the intersection is a conic counted twice. The equations of this conic are found to be

$$
\begin{gathered}
A\left(x_{3}-\lambda x_{8}\right)+B x_{4}=0, \\
A\left(L+2 \lambda+N \lambda^{2}\right) x_{3}{ }^{2}+2 B \lambda s x_{3} x_{4}+A C x_{4}{ }^{2}+2 A \lambda^{2} x_{1} x_{4}=0 .
\end{gathered}
$$

Case II (c). If $L+2 \lambda+N \lambda^{2}=0, A\left(C+E \lambda^{2}\right)-B^{2} \neq 0$, the characteristic is [(22)], and the intersection is three generators, one counted twice. The generator that is counted twice is the line $x_{4}=x_{3}-\lambda x_{2}=0$, which is tangent to the curve $C_{\lambda}$ at $P_{x}$. The other two generators are the lines whose equations are as follows:

$$
\begin{aligned}
& A \lambda\left(x_{3}-\lambda x_{2}\right)+\left[B \lambda \pm\left(B^{2}-A\left(C+E \lambda^{2}\right)\right)^{1 / 2}\right] x_{4}=0, \\
& A \lambda\left(C x_{4}+2 \lambda^{2} x_{1}\right)-\left[B \lambda^{2} s \mp\left(B^{2}-A\left(C+E \lambda^{2}\right)\right)^{1 / 2}\right] x_{3}=0,
\end{aligned}
$$

where the upper signs in the two equations are paired to give one generator and the lower signs the other.

Case II (d). If now in II(c) we let $A\left(C+E \lambda^{2}\right)-B^{2}=0$, then the characteristic is [(211)], and the intersection is two intersecting generators, each counted twice. The equations of these generators are respectively

$$
\begin{aligned}
x_{4} & =x_{3}-\lambda x_{2}=0, \\
A\left(x_{3}-\lambda x_{2}\right)+B x_{4} & =2 A \lambda^{2} x_{1}+2 B \lambda s x_{3}+A C x_{4}=0 .
\end{aligned}
$$


From the above results we note the following theorem:

At each point of an asymptotic curve on the net the intersection of the osculating quadrics degenerates completely into generators.

Case III. Now let $A=0, B \neq 0$. The characteristic is then [(31)], and the intersection is two intersecting generators, the tangents of our net at $P_{x}$, and a residual conic. We thus obtain the following result by using (22):

If the ray and axis are not reciprocal polar lines with respect to the osculating quadric $Q_{u}$, and if the curve $C_{\lambda}$ belongs to the associate conjugate net $(A=0)$, then the quadrics $Q_{u}$ and $Q_{v}$ intersect in the tangents of the net and a residual nondegenerate conic. The equations of this conic are

$$
\begin{gathered}
2 D \lambda^{2} x_{2}-2 B x_{3}-\left(C+E \lambda^{2}\right) x_{4}=0, \\
C D x_{4}^{2}+\left[2 B D-s\left(C+E \lambda^{2}\right)\right] x_{3} x_{4}-2 B s x_{3}^{2}+2 D \lambda^{2} x_{1} x_{4}=0 .
\end{gathered}
$$

Case IV. If $A=B=0, C+E \lambda^{2} \neq 0$, we find that the characteristic is [(211)], and the intersection is composed of the tangents of the net counted twice. The quadrics touch at each point of each tangent.

Case V. If $A=B=C+E \lambda^{2}=0$, the characteristic is [(1111)], and the two quadrics $Q_{u}$ and $Q_{v}$ coincide. Their equations both become

$$
C x_{4}^{2}+2 \lambda^{2} x_{1} x_{4}-2 \lambda r x_{2} x_{3}=0 .
$$

This completes the discussion of all cases that arise.

7. Conjugate nets. Let us now consider two curves $C_{\lambda}$ and $C_{\mu}$ on our net, and let us suppose that they are respectively embedded in two conjugate one-parameter families of curves on the net. By equation (8) we see that a necessary and sufficient condition for this is $\mu=-r / s$. Let $P_{x}$ denote the point where $C_{\lambda}$ crosses $C_{\mu}$. We shall denote by $Q_{u \lambda}$ the osculating quadric $Q_{u}$ of $C_{\lambda}$ at $P_{x}$ and by $Q_{u \mu}$ the osculating quadric $Q_{u}$ of $C_{\mu}$ at $P_{x}$. The equation of $Q_{u \mu}$ may be obtained from that of $Q_{u \lambda}$ simply by replacing $\lambda$ by $-r / s$. The equations of the osculating quadrics $Q_{u \lambda}$ and $Q_{u \mu}$ are thus as follows:

$$
\begin{array}{r}
A x_{3}{ }^{2}+2 B x_{3} x_{4}+C x_{4}{ }^{2}+2 \lambda^{2} x_{1} x_{4}-2 \lambda r x_{2} x_{3}=0 \\
A(1-L N) x_{3}{ }^{2}+2 H x_{3} x_{4}+K x_{4}{ }^{2}+2 r^{2} x_{1} x_{4}-2 \lambda r(1-L N) x_{2} x_{3}=0
\end{array}
$$

where the first of these is the same as the first of equations (18) and $H$ and $K$ are defined by

$$
\begin{aligned}
H= & r^{2} \mathscr{B}-r s\left(\mathcal{S}^{\prime}+s^{2}\left[L\left(r s^{\prime}-r^{\prime} s\right)\right.\right. \\
& \left.+r\left(L_{u} s-L_{v} r\right)\right] /[2 \lambda s(L N-1)], \\
K= & r\left\{\pi r-r F^{\prime}-s G-(r s k) /[\lambda(L N-1)]\right\} .
\end{aligned}
$$


The cone projecting the curve of intersection of the two quadrics (32) from the second vertex of the tetrahedron of reference is given by

$$
\begin{aligned}
2[B(1-L N)-\dot{H}] x_{3} x_{4}+[C(1-L N) & -K] x_{4}{ }^{2} \\
& +2\left[\lambda^{2}(1-L N)-r^{2}\right] x_{1} x_{4}=0 .
\end{aligned}
$$

This cone evidently consists of two planes, one of which is the tangent plane, $x_{4}=0$. But the tangent plane intersects each of the quadrics (32) in the two lines

$$
\begin{aligned}
& x_{3}=x_{4}=0, \\
& x_{4}=A x_{3}-2 \lambda r x_{2}=0 .
\end{aligned}
$$

Hence we draw the following conclusion:

At any point on the net the osculating quadric $Q_{u \lambda}$ of a curve of one family of a conjugate net always intersects the corresponding quadric $Q_{u \mu}$ of the curve of the other family in two conics, one of which is degenerate and is represented by equations (34). The other conic lies in the plane

$$
\text { (35) } 2 L\left(L+2 \lambda+N \lambda^{2}\right) x_{1}-2[B(1-L N)-H] x_{3}-[C(1-L N)-K] x_{4}=0 \text {. }
$$

Let us note that since $C_{\lambda}$ and $C_{\mu}$ are assumed distinct it follows as a consequence that $L+2 \lambda+N \lambda^{2} \neq 0$, and previous restrictions insure that $L \lambda r \neq 0$. Now let us project the curve of intersection of the quadrics (32) from the first vertex, $P_{x}$, of the tetrahedron of reference. The equation of the projecting cone is

$$
\begin{aligned}
& A\left[r^{2}-\lambda^{2}(1-L N)\right] x_{3}{ }^{2}+2\left(r^{2} B-\lambda^{2} H\right) x_{3} x_{4}+\{[L \lambda r(L+2 \lambda \\
& \left.\left.\left.+N \lambda^{2}\right) W^{(u)}\right] /(1-L N)\right\} x_{4}{ }^{2}-2 \lambda r\left[r^{2}-\lambda^{2}(1-L N)\right] x_{2} x_{3}=0 .
\end{aligned}
$$

Evidently the lines (34) are generators of this cone; so this is really the cone projecting from $P_{x}$ the conic lying in the plane (35), which does not pass through $P_{x}$. Hence the condition for the cone (36) to degenerate is just the condition for the residual conic to degenerate. But evidently $W^{(u)}=0$ is necessary and sufficient for this, and, as Green has shown, this is just the condition that insures that the $u$-tangents of the net form a $W$-congruence; that is, that the asymptotic curves on the two focal sheets of the congruence correspond. Hence we have proved the following theorem:

$A$ necessary and sufficient condition that the congruence of tangents to the curves $C_{u}$ of the net be generators of a $W$-congruence is that the residual conic of intersection of the two quadrics $Q_{u \lambda}$ and $Q_{u \mu}$ degenerate at every point of the net.

We have already seen that if the curve $C_{\mu}$ is embedded in a one-parameter family of curves that is conjugate to the family to which $C_{\lambda}$ belongs, then the 
osculating quadrics $Q_{u \lambda}$ and $Q_{u \mu}$ each intersect the tangent plane, $x_{4}=0$, in the lines (34); that is, in a $u$-tangent and a residual line. Now let us suppose that $C_{\lambda}$ and $C_{\mu}$ are any two curves which are respectively members of two distinct one-parameter families of curves, neither of which coincides with either family of the net itself. Then the corresponding osculating quadrics $Q_{u}$ for these two curves ordinarily will intersect the tangent plane in the $u$-tangent, $x_{3}=x_{4}=0$, and the two residual lines

$$
\begin{aligned}
& x_{4}=\left(L-N \lambda^{2}\right) x_{3}-2 \lambda(L+\lambda) x_{2}=0, \\
& x_{4}=\left(L-N \mu^{2}\right) x_{3}-2 \mu(L+\mu) x_{2}=0 .
\end{aligned}
$$

Now if we demand that these two residual lines coincide we get $\mu=-r / s$. Hence we draw the following conclusion:

$A$ necessary and sufficient condition that a net on our surface be a conjugate net is that the residual intersections (37) of the quadrics $Q_{u \lambda}$ and $Q_{u \mu}$ with the tangent plane coincide at every point of the net.

8. Metric considerations. It is the purpose of this section to indicate a method of studying the analogue of the preceding problem in metric threespace and to give a few results that have been obtained by the author for the metric situation. Throughout this section we shall employ the notation and vector methods of Blaschke* for studying the geometry of a surface in ordinary space.

The equations of the osculating quadrics at the point $x$ of a curve on the surface are here referred to a local orthogonal cartesian coordinate system, which is defined by the convention that any point $X$ given by an expression of the form

$$
X-x=y_{1} x_{u} / E^{1 / 2}+y_{2} x_{v} / G^{1 / 2}+y_{3} \xi
$$

where $\xi$ is the unit normal at the point $x$, shall have local coordinates $y_{1}$, $y_{2}, y_{3}$.

If we now let the partial differential equations of Gauss and Weingarten play the role of equations (1) and compute the equation of $Q_{u}$ in a manner similar to that of $\S 3$, we find that the equation of $Q_{u}$ is

$$
\begin{aligned}
E^{1 / 2}\left(L-N \lambda^{2}\right) y_{2}{ }^{2}+S G E^{1 / 2} y_{3}{ }^{2}-2 Q G^{1 / 2} y_{1} y_{2} \\
+2 P G y_{1} y_{3}+2 S(E G)^{1 / 2} y_{2} y_{3}+2 \lambda G E^{1 / 2} y_{3}=0
\end{aligned}
$$

where

* W. Blaschke, Vorlesungen über Differentialgeometrie, 3d edition, vol. 1, Berlin, 1930, pp. 85-118. 


$$
\begin{aligned}
P= & \left\{\begin{array}{c}
11 \\
2
\end{array}\right\}+\lambda\left\{\begin{array}{c}
12 \\
2
\end{array}\right\}, Q=L+M \lambda \\
\lambda Q S= & \left\{\begin{array}{c}
11 \\
2
\end{array}\right\}^{2}-\lambda^{\prime}\left\{\begin{array}{c}
11 \\
2
\end{array}\right\}+\lambda\left(2\left\{\begin{array}{c}
11 \\
2
\end{array}\right\}\left\{\begin{array}{c}
12 \\
2
\end{array}\right\}-\left\{\begin{array}{c}
11 \\
1
\end{array}\right\}\left\{\begin{array}{c}
11 \\
2
\end{array}\right\}+\left\{\begin{array}{c}
11 \\
2
\end{array}\right\}_{u}\right. \\
& \left.+L(F L-E M) / W^{2}\right)+\lambda^{2}\left(\begin{array}{c}
11 \\
2
\end{array}\right\}\left\{\begin{array}{c}
22 \\
2
\end{array}\right\}-2\left\{\begin{array}{c}
11 \\
2
\end{array}\right\}\left\{\begin{array}{c}
12 \\
1
\end{array}\right\}+2\left\{\begin{array}{c}
11 \\
2
\end{array}\right\} \\
& \left.+2 L(F M-E N) / W^{2}\right)+\lambda^{3}\left(\left\{\begin{array}{c}
11 \\
2
\end{array}\right\}\left\{\begin{array}{c}
22 \\
1
\end{array}\right\}-2\left\{\begin{array}{c}
12 \\
1
\end{array}\right\}\left\{\begin{array}{c}
12 \\
2
\end{array}\right\}+\left\{\begin{array}{c}
22 \\
2
\end{array}\right\}_{u}\right. \\
& \left.+N(F L-E M) / W^{2}\right), \\
2 \lambda Q T= & L \lambda^{\prime}-2 L\left\{\begin{array}{c}
11 \\
2
\end{array}\right\}+\lambda\left(L\left\{\begin{array}{c}
11 \\
1
\end{array}\right\}-L\left\{\begin{array}{c}
12 \\
2
\end{array}\right\}-2 M\left\{\begin{array}{c}
11 \\
2
\end{array}\right\}-L_{u}\right) \\
& +\lambda^{2}\left(L\left\{\begin{array}{c}
22 \\
2
\end{array}\right\}+2 L\left\{\begin{array}{c}
12 \\
1
\end{array}\right\}-N\left\{\begin{array}{c}
11 \\
2
\end{array}\right\}-2 L_{v}\right) \\
& +\lambda^{3}\left(N\left\{\begin{array}{c}
12 \\
2
\end{array}\right\}+2 M\left\{\begin{array}{c}
12 \\
1
\end{array}\right\}-L\left\{\begin{array}{c}
22 \\
1
\end{array}\right\}-N_{u}\right) .
\end{aligned}
$$

The equation of the osculating quadric $Q_{v}$ may now be written down by symmetry, or it may be obtained by direct calculation.

The discriminant $\Delta$ of the quadric (39) is

$$
\Delta=\lambda^{2} E G^{3} Q^{2} \neq 0,
$$

for $E, G$ are positive on a real surface, $\lambda \neq 0$ since $C_{\lambda}$ was assumed not a $u$ curve, and $Q \neq 0$ since $C_{\lambda}$ was supposed not a curve of the family conjugate to the family of $u$-curves of the net. Thus the osculating quadric $Q_{u}$ is always nonsingular for a real analytic surface.

If we simplify the analysis by taking the parametric net to be orthogonal we get the following interesting result. The determinant $D^{\prime}$ of the matrix of the second degree terms in the resulting equation of the quadric $Q_{u}$ then becomes

$$
D^{\prime}=\left(E^{1 / 2} / \lambda\right)\left(A_{0} \lambda^{\prime}+A_{1}+A_{2} \lambda+A_{3} \lambda^{2}+A_{4} \lambda^{3}\right)
$$

wherein

$$
\begin{aligned}
& A_{0}=-G\left(L G_{u}+M E_{v}\right) / 2, \\
& A_{1}=\left(4 L^{2} M G+2 L G E_{u v}+M E_{v}{ }^{2}-L E_{v} G_{u}-2 L_{u} E_{v} G\right) / 4,
\end{aligned}
$$




$$
\begin{aligned}
A_{2}= & \left(2 G L_{u} G_{u}+8 L^{2} N G+4 L G E_{v v}-2 L E_{v} G_{v}-M G E_{u} E_{v} / E+4 L M^{2} G\right. \\
& \left.+2 N E_{v}^{2}+2 M G E_{u v}-2 E_{v} G_{u}-4 G L_{v} E_{v}-L G E_{u} G_{u} / E\right) / 4 \\
A_{3}= & \left(12 L M N G-2 L G G_{u v}+4 M G E_{v v}-3 M E_{v} G_{v}-2 G N_{u} E_{v}+L G_{u} G_{v}\right. \\
& \left.-2 N E_{v} G_{u}\right) / 4 \\
A_{4}= & \left(4 M^{2} N G-M G E_{v} G_{u} / E-2 M G G_{u v}+2 M G_{u} G_{v}-L G G_{u}{ }^{2} / E+2 G N_{u} G_{u}\right) / 4
\end{aligned}
$$

Now suppose $C_{\lambda}$ is a curve such that $A_{0} \neq 0$. Since by definition the rulings of the quadric $Q_{u}$ are real, we find that the osculating quadric $Q_{u}$ at every point of a curve $C_{\lambda}$ on an orthogonal net is a hyperbolic paraboloid if, and only if, $C_{\lambda}$ belongs to the family of hypergeodesics defined by $D^{\prime}=0$. Otherwise $Q_{u}$ is a hyperboloid.

Lambuth College, Jackson, Tenn. 